\title{
MR Directed Focal Hyperthermia for Pelvic Disease
}

Final Progress Report

December 8, 2014

\section{PI - Chris J Diederich, PhD}

Vasant Salgaonkar, PhD, Viola Rieke, PhD, Eugene Ozhinsky, PhD, John Kurhanewicz, PhD, and I.C. Joe Hsu, MD, University of California, San Francisco

\section{Summary of Final Quarter}

The overall objective of this research project was to devise the required techniques and evaluate feasibility of delivering targeted prostate hyperthermia using the Insightec ExAblate Prostate Ablation System. Our motivation is driven by the possibility that this MR guided platform, as already approved for prostate ablation, could be modified beyond the intended scope of use as a means to fast-track implementation of MR guided prostate hyperthermia as an adjunct to radiation therapy, targeted drug delivery, or immunotherapy. Earlier progress reports described our efforts developing theoretical simulations and beam forming techniques specific and limited by the intricacies of the hardware and software controlling the Insightec prostate system. Subsequent patient specific biothermal and acoustic simulations, representative of targeting various regions of the prostate with hyperthermia, were performed to demonstrate feasibility. Further, communication protocols and beam forming illumination patterns specific to hyperthermia with this array layout/system were developed, implemented, and evaluated in thermal dosimetry experiments in tissue mimicking phantoms to demonstrate feasibility. The phantom experiments were monitored using custom multi-slice MRTI which is concurrently under development for monitoring of hyperthermia treatments with this device and system. Over the final quarter we have devoted most of our efforts towards a more seamless integration of the system control, implementation of PI control with TTL direct gated control of the ExAblate system through the CPC unit to modulate power, and integration of feedback control loop with MRTI using selected ROI to control power applied during 15-20 therapy application. This complete "system" was evaluated in multiple in vivo heating trials in porcine muscle tissue as to demonstrate successful preliminary implementation. Some key results are summarized below each aim below, along with a listing of presentations and publications.

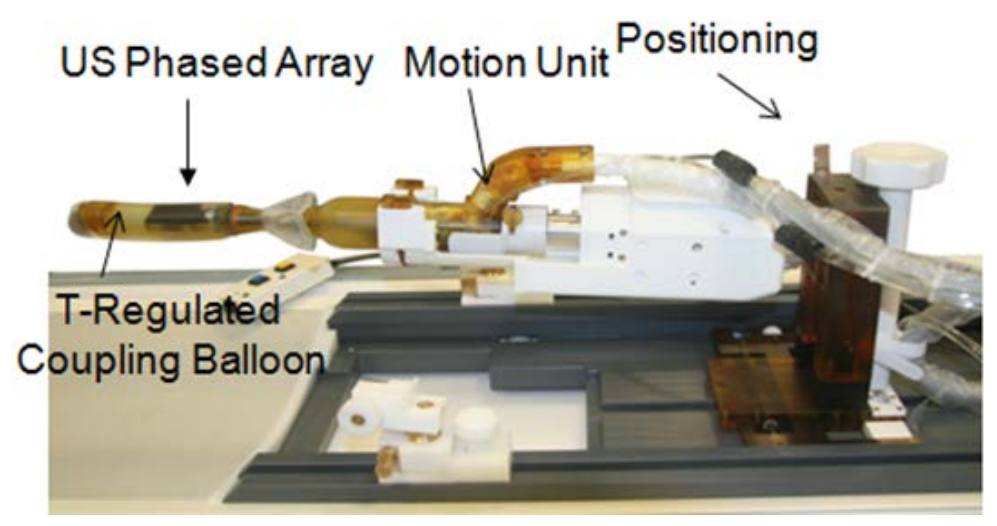

Fig. 1. Insightec ExAblate Prostate array for MR guided thermal ablation of prostate cancer. This system is designed for short duration and focal ablation of multiple small regions in the prostate. In this work we determine and implement methods, working within the hardware and software constraints of the system, to produce larger regions of hyperthermia for $15 \mathrm{~min}$ 
AIM 1 Implement operational modifications and integrate custom multi-slice or volumetric MR temperature guidance and system control as required to utilize the InSightec 2100 ER array to deliver targeted and long-duration continuous hyperthermia (versus pulsed hyperthermia).

We have worked closely with Insightec to establish the existing system constraints (imposed by original design for producing and monitoring short, tightly focused sonications specific to ablation) and devised communication protocols to control the Insightec Prostate phased array through the CPC interface. During our interactions we were able to work with the engineers to determine some parameters such as maximum allowable power levels and duration of $\mathrm{CW}$ application, and have now applied these values to our control algorithms. In this fashion, we can modify the applied surface intensity, individual or customized complex phase patterns, sequence duration, position regeneration, phasing patterns, and other applicator parameters as demonstrated to allow protracted or long duration hyperthermia over a large contiguous volume. Custom multi-slice MRTI using RTHawk was developed for this application and implemented to monitor the hyperthermia temperature elevations over the longer intervals required. It also includes a PI controller with MRTI based temperature feedback loop with user defined ROI to apply feedback control of applied power to maintain hyperthermia temperatures.

\section{Implementing MRgHT}

Generation of Beamforming Patterns: Array Specific (layout, power \& phasing limits)
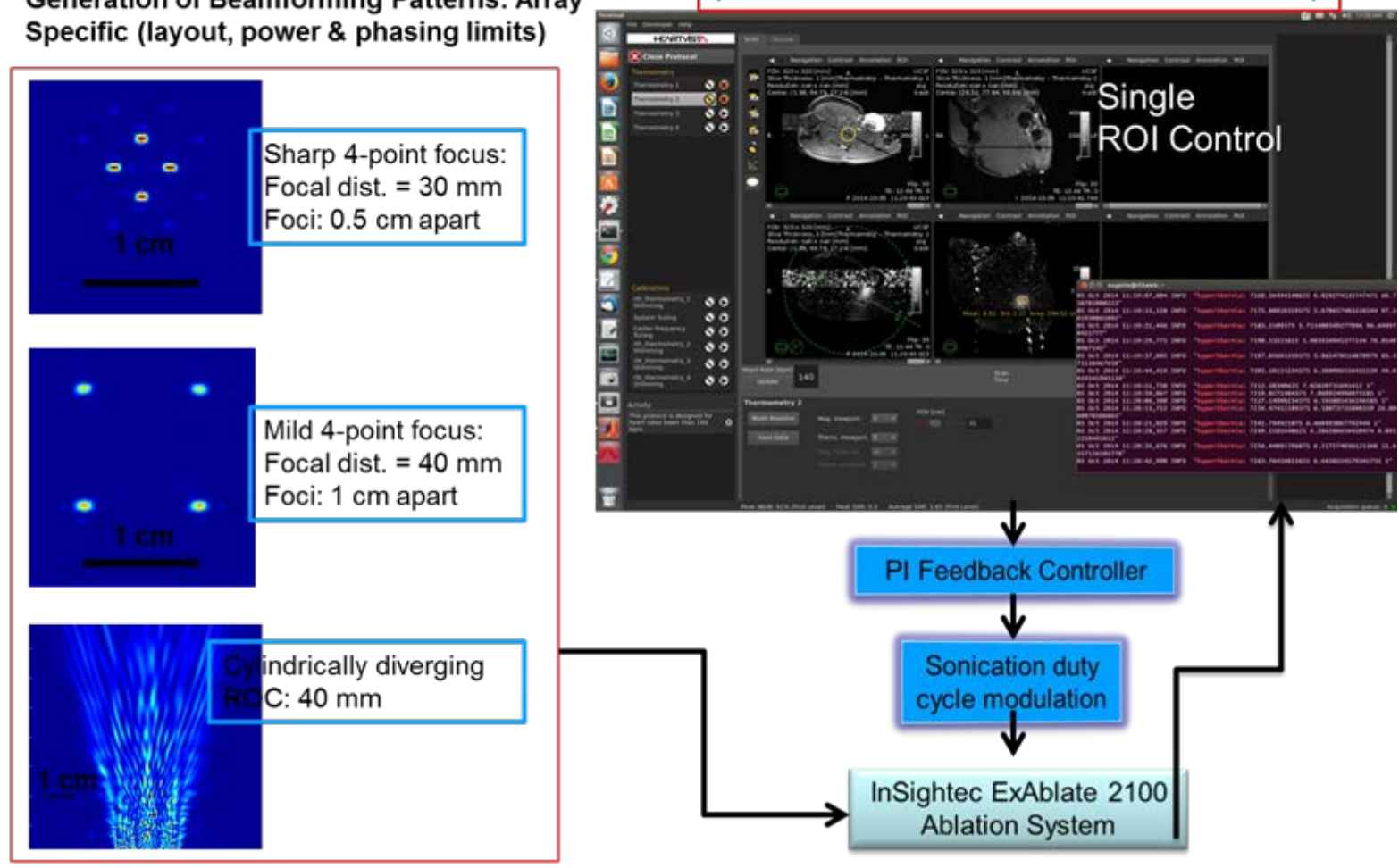

Fig. 2. Overall flow chart schematic of control and delivery schema to produce targeted, long duration or protracted hyperthermia, with ExAblate prostate ablation array and system; hyperthermia sonication pattern selection, power application and illumination pattern synthesis, MRTI for hyperthermia monitoring including ROI real time temperature based feedback control of applied power for long durations. 


\section{AIM 2 Design and evaluate treatment delivery strategies using patient-specific simulations.}

3D patient specific models were created, selected to bracket anticipated clinical treatment sites. MR images that clearly delineate focal disease were obtained and used to generate 3D biothermal models, together with complex acoustic field calculations within the prostate and defined intraprostatic lesions. The acoustic simulations were specific to the Insightec prostate array layout and wiring (this information was given to us on a confidential and proprietary basis). Determination and assessment of various phasing patterns for generating conformal and effective hyperthermia for various locations and dimensions of tumor target were performed. General phasing patterns, with either multipoint single focus, diverging, converging, and planar static patterns were investigated, as well multi-point electronic scanning. PI feedback control algorithms were developed for controlling applied power levels based upon selected ROI region within heated field. Together, these theoretical studies clearly demonstrated feasibility of this approach and potential for implementation in human studies - a summary of applied patterns and approximate treatment volumes are shown in Table 1. (See attached publications for details).

\section{Targeted HT w/ Multi-Focal Patterns}

Specific proprietary array and hardware parameters modeled
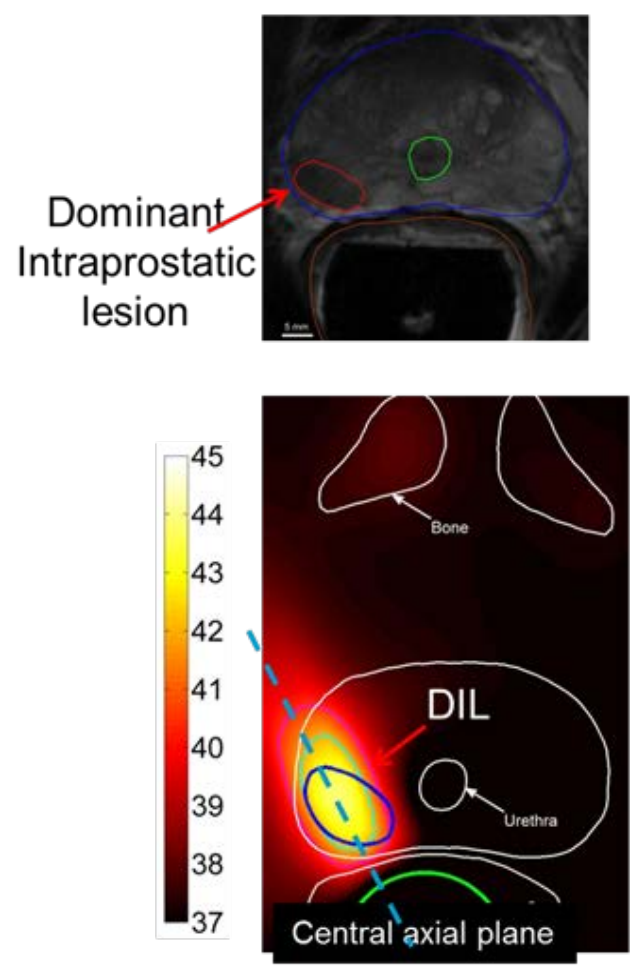

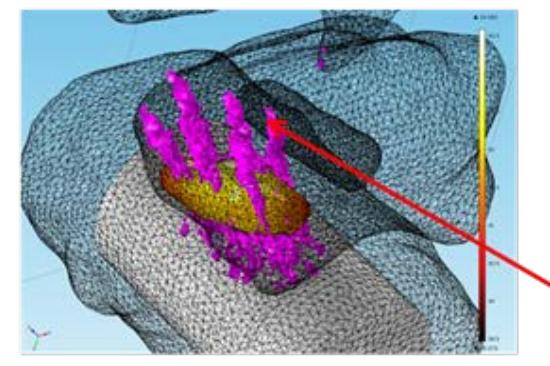

Simultaneous

6-point focus

$\mathrm{SAR}=300 \mathrm{~W} / \mathrm{kg}$

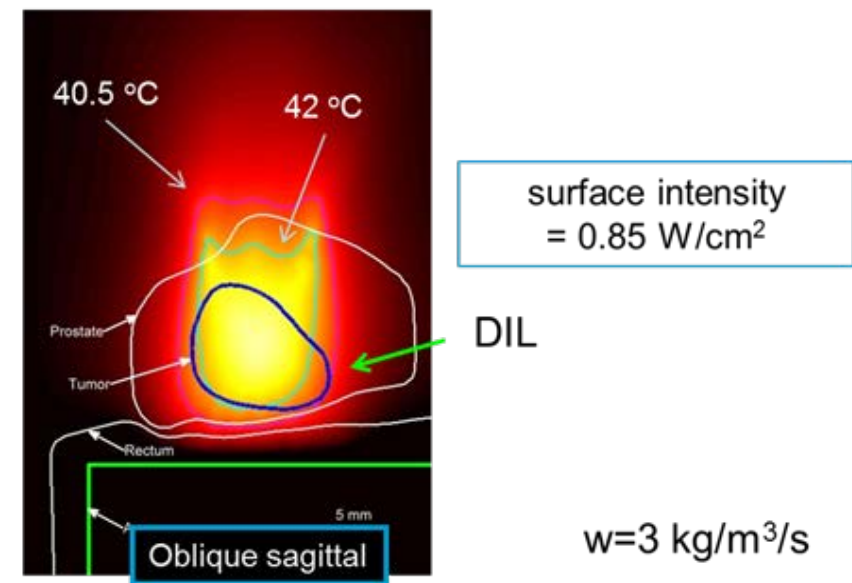

Fig. 3. Example cases of simulation studies demonstrating selection of DIL for targeting, generation of acoustic beam patterns to best fit the entire tumor target regions, and application of beam patterns at power levels sufficient to generate hyperthermia for times greater than 15 min duration. 


\begin{tabular}{|c|c|c|c|c|}
\hline Beamforming & $\begin{array}{l}\text { Surface } \\
\text { Intensity } \\
\left(\mathbf{W} / \mathrm{cm}^{2}\right)\end{array}$ & $\begin{array}{l}\text { Vol. } \mathrm{T}>40^{\circ} \mathrm{C} \\
\left(\mathrm{cm}^{3}\right)\end{array}$ & $\begin{array}{c}\text { Vol. } \mathrm{T}>41^{\circ} \mathrm{C} \\
\left(\mathrm{cm}^{3}\right)\end{array}$ & $\begin{array}{l}\text { Targeting capability and } \\
\text { caveats }\end{array}$ \\
\hline $\begin{array}{l}\text { Simultaneous multi- } \\
\text { point focusing } \\
\text { (Num of foci }=4-8 \text { ) }\end{array}$ & $0.68-0.72$ & $8.8-10.6$ & $4.5-5.8$ & $\begin{array}{l}\text { Selective heating of small targets. } \\
\text { Potential unwanted hotspots due to side }\end{array}$ \\
\hline $\begin{array}{l}\text { Curvilinear focusing } \\
\text { (depth }=25-35 \mathrm{~mm} \text { ) }\end{array}$ & $0.7-0.75$ & $12-12.1$ & $6.0-6.6$ & Useful for tailored conformal HT \\
\hline $\begin{array}{l}\text { Diverging sonication } \\
\text { (radius of curvature = } \\
10-40 \mathrm{~mm} \text { ) }\end{array}$ & $1.05-1.2$ & $24.4-37.2$ & $13.3-21.5$ & $\begin{array}{l}\text { Uniform energy deposition. Suitable for } \\
\text { targeting posterior quadrants and hemi- } \\
\text { gland targets. Potential bone heating.. }\end{array}$ \\
\hline Planar sonication & 0.94 & 35 & 22.8 & \\
\hline
\end{tabular}

Fig. 4. Summary of parametric patient specific studies demonstrating targeting capabilities and volumes treatable with various illumination patterns - all within the capabilities of the modified system.

AIM 3 Validate and characterize system performance within tissue phantoms and in vivo models. The modified ER ablation system and preliminary controlling software, along with calculated phasing patterns (from Aim 2) for a variety of tumor shapes and target localization were evaluated in static tissue mimicking phantom within the MR using multislice MRTIRTHawk to setup and monitor temperature (Fig. 5). These experiments demonstrated hyperthermia delivery can be performed, albeit in a non-perfused environment. As a final verification of the feasibility of this approach, targeted heating exposures were performed in

\section{Experiment and Comparison to Simulations}
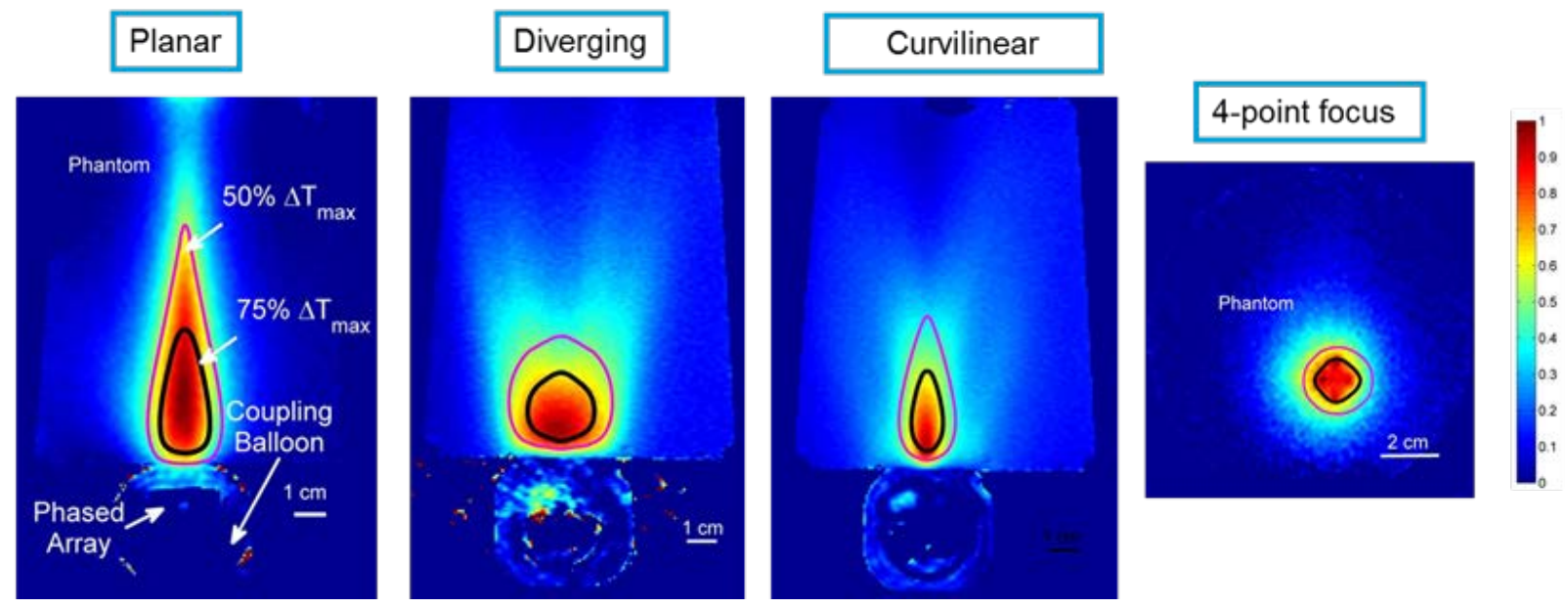

Fig. 5. Validation of phasing pattern and array control to generate hyperthermia patterns in tissue mimicking phantom under MRTI. The theoretical determinations were compared and were in with excellent agreement with MRTI (Salgaonkar et al. 2014). 
multiple trials using the porcine thigh muscle in vivo (Fig. 6). The ExAblate array was positioned at sites on the inner thigh, and various sonication patterns were applied to deliver large volume long duration hyperthermia using the planar or cylindrical diverging patterns, or for single or multiple focal patterns for smaller targets. The PI temperature based feedback controller with TTL actuation at the CPC panel was used to control applied power in real time, based upon duty-cycle control of the output. The position and dimensions of the ROI for temperature control could be varied on the MRTI platform. Therapeutic temperature elevations of $>15$ min duration could be achieved in all cases tested, using maximum $\mathrm{CW}$ power levels well below the maximum threshold.

\section{MRg Feedback Controlled Hyperthermia In Vivo}

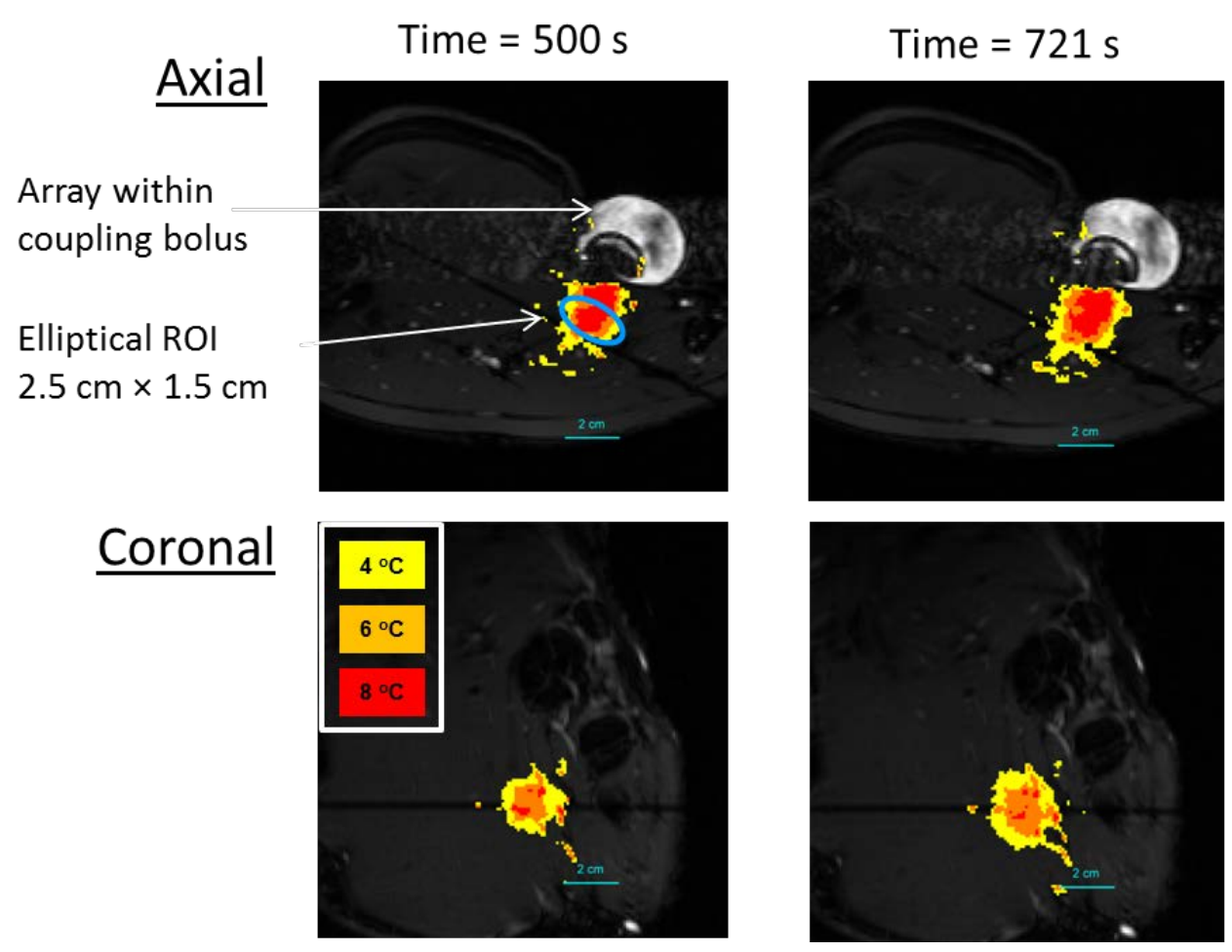

Fig. 6. Example in vivo thermal dosimetry of hyperthermia applied with the Insightec ExAblate Prostate Ablation system to the porcine thigh muscle using a cylindrically diverging array illumination pattern and continuous heating under MRTI feedback control for 15 min duration. Multislice MRTI in coronal and axial slices are shown. 


\section{Publications}

- Salgaonkar VA, Prakash P, Rieke V, Ozhinsky E, Plata J, Kurhanewicz J, Hsu IC, Diederich CJ. Model-based feasibility assessment and evaluation of prostate hyperthermia with a commercial MR-guided endorectal HIFU ablation array. Medical Physics Med Phys. 2014 Mar;41(3): PMID: 24593742

- Salgaonkar VA, Prakash P, Plata J, Holbrook A, Rieke V, Kurhanewicz J, Hsu I-C, Diederich CJ. Targeted hyperthermia in prostate with an MR-guided endorectal ultrasound phased array: patient specific modeling and preliminary experiments. Proc SPIE 85842013.

\section{Abstracts/Oral Presentations}

- V.A. Salgaonkar, P. Prakash, V. Rieke, E. Ozhinsky, J. Kurhanewicz, J.C. Plata, I. Hsu, C. Diederich, Implementation of sonication and feedback control strategies for targeted hyperthermia in prostate with a commercial MR-guided endorectal ultrasound ablation array, FUS Symposium, Bethesda, MD, 2014

- Eugene Ozhinsky, Vasant A. Salgaonkar, Chris J. Diederich, and Viola Rieke, MR-Guided Prostate Hyperthermia with a Commercial Endorectal HIFU Ablation Array, ISMRM 2014

- V.A. Salgaonkar, P. Prakash, V. Rieke, E. Ozhinsky, J. Kurhanewicz, J.C. Plata, I. Hsu, C. Diederich, Model-based feasibility assessment and evaluation of prostate hyperthermia with a commercial MR-guided endorectal HIFU ablation array, ISTU 2014

- Salgaonkar VA, Rieke V, Ozhinsky E, Prakash P, Plata J, Kurhanewicz J, Hsu IC, Diederich CJ. Model-based Feasibility Assessment and Evaluation of Prostate Hyperthermia with a Commercial MR-guided Endorectal HIFU Ablation Array. Annual Meeting Acoustical Society of America, San Francisco, CA, USA Dec. 2013

- Salgaonkar VA, Rieke V, Ozhinsky E, Prakash P, Kurhanewicz J, Hsu IC, Diederich CJ. Model-based feasibility assessment and evaluation of prostate hyperthermia with a commercial MR-guided endorectal HIFU ablation array. $2^{\text {nd }}$ European Symposium on Focused Ultrasound Therapy. Rome, Italy,. Oct. 2013

- Salgaonkar VA, Rieke V, Chang A, Hsu IC, Kurhanewicz J, Scott S, Plata J, Diederich CJ. MR-guided endocavity ultrasound hyperthermia for treating pelvic malignancies: patient specific modelling and preliminary experiments. Annual Symposium Society for Thermal Medicine. Aruba, Apr. 2013.

- Salgaonkar VA, Prakash P, Plata J, Holbrook A, Rieke V, Kurhanewicz J, Hsu IC, Diederich CJ. Targeted hyperthermia in prostate with an MR-guided endorectal ultrasound phased array: patient specific modeling and preliminary experiments. Photonics West Symposium, SPIE, San Francisco CA, USA, Jan. 2013. 